\title{
Scaling of Wave-Packet Dynamics in an Intense Midinfrared Field
}

\author{
J. Tate, ${ }^{1}$ T. Auguste, ${ }^{2}$ H. G. Muller, ${ }^{3}$ P. Salières, ${ }^{2}$ P. Agostini, ${ }^{1}$ and L. F. DiMauro ${ }^{1}$ \\ ${ }^{1}$ Department of Physics, The Ohio State University, Columbus, Ohio 43210, USA \\ ${ }^{2}$ CEA, CE Saclay, 91191 Gif Sur Yvette, France \\ ${ }^{3}$ FOM AMOLF 407 Kruislaan, 1098 SJ Amsterdam, The Netherlands
}

(Received 28 April 2006; published 4 January 2007)

\begin{abstract}
A theoretical investigation is presented that examines the wavelength scaling from near-visible $(0.8 \mu \mathrm{m})$ to midinfrared $(2 \mu \mathrm{m})$ of the photoelectron distribution and high harmonics generated by a "single" atom in an intense electromagnetic field. The calculations use a numerical solution of the timedependent Schrödinger equation (TDSE) in argon and the strong-field approximation in helium. The scaling of electron energies $\left(\lambda^{2}\right)$, harmonic cutoff $\left(\lambda^{2}\right)$, and attochirp $\left(\lambda^{-1}\right)$ agree with classical mechanics, but it is found that, surprisingly, the harmonic yield follows a $\lambda^{-(5-6)}$ scaling at constant intensity. In addition, the TDSE results reveal an unexpected contribution from higher-order returns of the rescattering electron wave packet.
\end{abstract}

DOI: 10.1103/PhysRevLett.98.013901

PACS numbers: $42.65 . \mathrm{Ky}, 32.80 . \mathrm{Fb}, 32.80 . \mathrm{Rm}$

A method for producing bursts of high-energy photoelectrons and photons has its origin in the fundamental interaction of an "isolated" atom with an intense, lowfrequency (nonresonant) electromagnetic field. The foundations of these processes are based on scaling metrics [1] and quasiclassical physics [2,3] which are linked to a tunnel ionized continuum electronic wave packet being "coherently" driven back into the core by the intense oscillating field. It is not surprising that under these conditions the inherent characteristics of the intense optical field, such as amplitude, phase, duration and frequency, are intimately connected to the observables. Numerous investigations have exploited the utility of the field parameters, for example, to produce isolated attosecond pulses [4,5] or to realize the time domain analogy of the Young's double slit experiment [6]. However, the wavelength has been largely ignored due, in part, to the limitation imposed by ultrafast amplifier technology which confines experiments to near-visible wavelengths, e.g., $0.8 \mu \mathrm{m}$ for titanium sapphire media. Nonetheless, the above principles suggest that scaling the intense laser-atom interaction towards longer wavelength will result in the realization of more energetic particles, many-body interactions and shorter bursts of attosecond ( 1 as $\equiv 10^{-18}$ s) light. Furthermore, the impetus for understanding the fundamental scaling is also motivated by recent advances in new ultrafast amplifier engineering [7] that will provide the necessary experimental tools.

In this Letter, a theoretical investigation is presented that explores the fundamental wavelength scaling of the intense laser-atom interaction. The primary focus is to map the evolving isolated quantum response and the corresponding relationship to the classical behavior as the wavelength is varied from the near-visible to the midinfrared $(0.8-2 \mu \mathrm{m})$. Both numerical solutions of the timedependent Schrödinger equation (TDSE) within a singleactive electron approximation (SAE) [8] and a strong-field approximation (SFA) $[9,10]$ are used. The scaling is inves- tigated using argon and helium since (i) they are prototypical laboratory atoms, (ii) computationally efficient model potentials exist for TDSE calculations [11], and (iii) the prescribed wavelength range results in a dramatic alteration in the wave packet dynamics.

Scaling laws are central to physics and analogous principles can be derived for the intense laser-atom interaction. First, the energy emitted as electrons and photons is defined by the ponderomotive energy, $U_{p}$, given in atomic units as $I / 4 \omega^{2}$, where $I$ and $\omega$ are the laser intensity and frequency, respectively. The maximum energy for electrons and photons are $10 U_{p}$ and $3.17 U_{p}+I_{p}$, respectively, where $I_{p}$ is the ionization potential. Thus, at constant intensity, longer wavelengths generate more energetic particles, opening the possibility of producing multikilovolt electrons and coherent harmonic radiation. Second, the evolution of the quivering wave packet reflects the initial conditions of the excitation mode as described by the Keldysh parameter [1] $\gamma=\sqrt{I_{p} / 2 U_{p}}$ that separates the tunneling $(\gamma \ll 1)$ and multiphoton $(\gamma \gg 1)$ regimes. For an atom at constant intensity $\gamma$ scales as $\omega$, implying that longer wavelengths drive the interaction further into the tunnel regime and therefore closer to the semiclassical behavior. Third, the formation of asec pulses depends on the emission time of the various harmonic frequency components (harmonic orders) contributing to the frequency comb or plateau. Depending on the trajectory of the wave packet, the high frequency components either precede or lag behind the low-frequency components and the asec burst has either a negative or positive chirp, respectively, which limits the attosecond pulses from high harmonics [12]. A simple argument predicts the dependence of the chirp on the fundamental wavelength: the propagation time of the electron in the continuum, and thus the emission time of successive harmonics, is proportional to the fundamental laser period $T$, while the harmonic energy is proportional to $U_{p}$. Thus the absolute value of the chirp 
(the 2nd-order spectral phase) scales as $\beta \propto T / U_{p} \propto$ $(I \lambda)^{-1}$ and is inversely proportional to $\lambda$. As a function of the bandwidth $B$, the pulse duration $\tau$ can be written as $\tau \propto \sqrt{\left(1+\beta^{2} B^{4}\right)} / B$. The first term is the time-frequency Fourier relationship while the second describes the effect of the chirp. The minimum duration $\tau_{m}$ is given by $\tau_{m} \propto$ $\sqrt{2 \beta} \propto 1 / \sqrt{I \lambda}$, i.e., inversely proportional to the square root of the driving wavelength. Finally, since the wave packet propagation time in the continuum is proportional to the laser wavelength, the wave packet has more time to spread with increasing drive wavelength, thus diluting the efficiency of the rescattering process that produces energetic harmonics and electrons.

The thrust of this Letter is to understand the wavelength scaling at the quantum level for the single atom. These calculations will allow direct comparison to future gas phase photoelectron experiments. In the TDSE calculations the model argon atom, initially in the ground state, is treated in the SAE approximation with spin-orbit coupling neglected [11]. The atom is subjected to an $N$-cycle flat-top laser pulse described by the vector potential $A(t)=$ $A_{0} \hat{z} \cos \omega t(0 \leq t \leq N T)$; the pulse is switched "on" and "off" in a half cycle. The stability of the results is verified for both slower turn-on ramps and sine-squared pulse shapes. The code directly outputs the angle-resolved photoelectron energy distribution while the harmonic spectra were obtained from a Fourier transform of the timedependent acceleration [13]. The calculations were done for several wavelengths between 0.8 and $2 \mu \mathrm{m}$, and for a broad range of intensities.

Figure 1 shows the (a) angle-integrated photoelectron distributions in scaled energy and (b) harmonic spectra generated by an 8-cycle pulse in argon for two different wavelengths at $0.16 \mathrm{PW} / \mathrm{cm}^{2}$, corresponding to $U_{p}=$ 59.7 and $9.5 \mathrm{eV}$ for 2 and $0.8 \mu \mathrm{m}$, respectively. It is clear that the $2 \mu \mathrm{m}$ light generates much higher energy electrons $(\leq 600 \mathrm{eV})$ and photons $(210 \mathrm{eV}$ cutoff $)$ due to the $\lambda^{2}$ scaling of $U_{p}$. Achieving equivalent cutoffs at $0.8 \mu \mathrm{m}$ would require an intensity of $1 \mathrm{PW} / \mathrm{cm}^{2}$, which is well beyond the depletion of the argon ground state. The characteristic shape of the $2 \mu \mathrm{m}$ electron distribution, with an abrupt cutoff at $10 U_{p}$, is indicative of the semiclassical prediction for elastic rescattering experimentally demonstrated in helium [14]. However, the monotonically decaying $0.8 \mu \mathrm{m}$ photoelectron spectrum shows a significant contribution from multiphoton ionization. Similarly, the harmonic spectrum from argon at $2 \mu \mathrm{m}$ shows a sharp cutoff at the predicted value of $E_{\max }=3.2 U_{p}+I_{p}$, while the lower energy high harmonic spectrum from $0.8 \mu \mathrm{m}$ excitation shows a gradual decay near the expected cutoff. TDSE results from helium are very similar, and in fact show closer adherence to the semiclassical model even at $0.8 \mu \mathrm{m}$ due to the higher intensities necessary to achieve ionization and high harmonic generation (HHG).

In addition to the energy scaling, the HHG yield is an important fundamental quantity with practical implica-
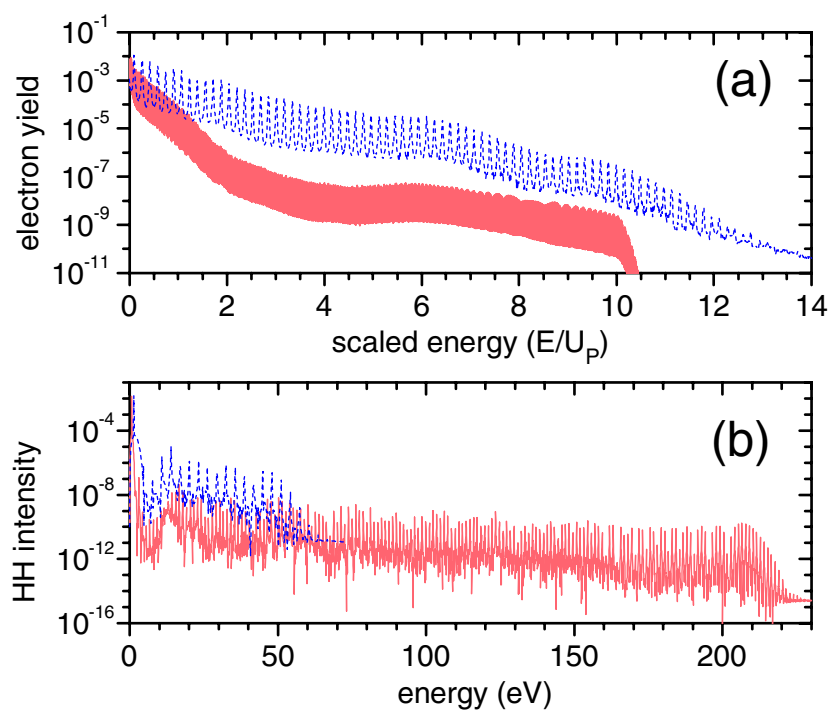

FIG. 1 (color online). (a) TDSE angle-integrated electron spectra for argon exposed to a flat-top 8-cycle pulse with intensity $0.16 \mathrm{PW} / \mathrm{cm}^{2}$ and wavelengths of 2 (solid line) and $0.8 \mu \mathrm{m}$ (dashed line). (b) Harmonic spectra for the same conditions.

tions. A previously stated $\lambda^{-3}$ scaling, supported by an experiment over a small wavelength range [15], has a simple physical interpretation: $\lambda^{-2}$ originates from the wave packet spread while an additional $\lambda^{-1}$ factor arises from the conversion from photon number to energy. However, this is only valid for a single harmonic with both $I_{p} / \omega$ and $U_{p} / \omega$ constant [10]. A more relevant experimental observable is the power spectrum at both constant $I_{p}$ and $I$. We use two independent methods to study the scaling of an average harmonic yield, obtained by integrating the power spectrum over a fixed bandwidth. TDSE calculations were performed in argon in the range 0.8-2.0 $\mu \mathrm{m}$. For helium, TDSE calculations become computationally intractable; instead we utilize the SFA method [9] including ground state depletion [16] over the wavelength range of $0.8-1.6 \mu \mathrm{m}$ (Fig. 2). In contrast to [17], our calculations, performed for low ionization, give a harmonic yield that scales $\approx \lambda^{-5.5 \pm 0.5}$ at constant $I$ and agree qualitatively with Ref. [10]. The physical origin of this faster dependence is not yet fully understood. Although such scaling is detrimental for HHG, it must be stressed that the longer wavelength light produces energetic particles that are otherwise absent at shorter wavelengths and for which the scaling breaks down.

Although individual dispersive effects (electronic, geometric, dipole phase) generally result in a more rapid decrease of the HHG scaling with increasing wavelength [15], control parameters (focusing and target geometry, density) enable a means for a global minimization. Simulations are in progress to investigate these conditions.

Many HHG observables have found qualitative explanations based on rescattering trajectories that involve only the first return (after $\sim 1 / 2$ optical period) of the electron to 


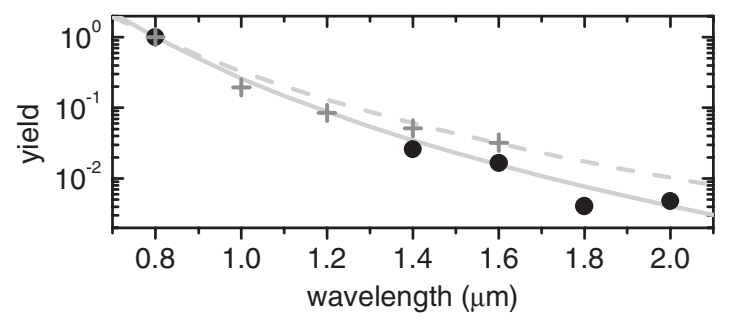

FIG. 2. The wavelength dependence of the integrated HHG yield at constant intensity for TDSE $(-)$ and SFA (+). The TDSE results for argon at $0.16 \mathrm{PW} / \mathrm{cm}^{2}$ are integrated from $20-50 \mathrm{eV}$. SFA results for helium at $0.445 \mathrm{PW} / \mathrm{cm}^{2}$ are integrated from 40-80 eV. The dashed and solid lines show a $\lambda^{-5}$ and $\lambda^{-6}$ dependence, respectively.

the ion core. The first return presents, in a time versus energy diagram, two branches, often referred to as the short $\left(\tau_{1}\right)$ and long $\left(\tau_{2}\right)$ trajectories, which coalesce at the maximum energy of $3.17 U_{p}$ [the physical origin of the cutoff in Fig. 1(b)]. The time dependence of these trajectories derived purely from classical mechanics are plotted as solid lines in Fig. 3. The two branches are distinguished by the sign of their chirp and their coherence time. However the classical model does allow for the possibility that the electron, rather than recombining during the first encounter, scatters from the core and returns a second $\left(\tau_{3}, \tau_{4}\right)$, third $\left(\tau_{5}, \tau_{6}\right)$ or $n$th $\left(\tau_{2 n-1}, \tau_{2 n}\right)$ time. Each higher-order return originates from the "long" trajectory and can also be defined by two branches. For $n \geq 2$ they have a different maximum kinetic energy $\leq 2.4 U_{p}$; and a decreasing

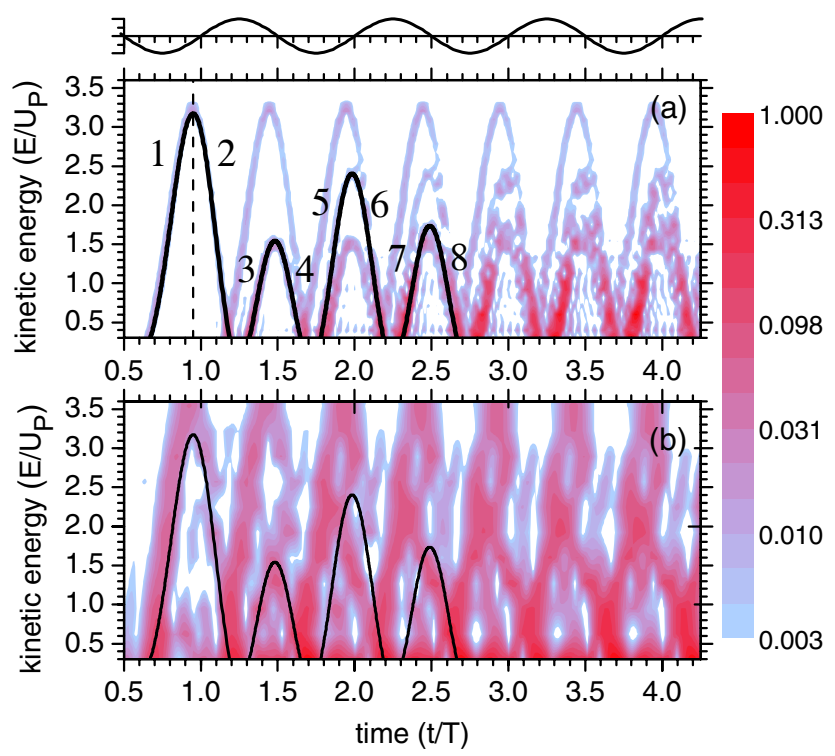

FIG. 3 (color online). TDSE time history of the HHG emission energy for argon excited by (a) 2 and (b) $0.8 \mu \mathrm{m}$ driving laser with intensity $0.16 \mathrm{PW} / \mathrm{cm}^{2}$. The solid black lines are the purely classical prediction for return energy. The numeric label corresponds to the $\tau_{n}$ trajectory. The top trace shows the electric field of the input pulse. effectiveness for HHG due to the wave packet spread is expected [18].

One of the main results of this Letter is that the TDSE analysis presented in Fig. 3 shows that higher-order returns, $n \geq 2$, play an unexpectedly important role in the HHG process. Figure 3 is a plot of the intensity of the emitted harmonics as a function of energy and time for flattop pulses at two different wavelengths. The plots are generated by first calculating the full harmonic spectrum, as illustrated in Fig. 1(b), then applying a rectangular spectral filter to select only a small bandwidth. The filtered region is inverse Fourier transformed to give the harmonic intensity as a function of time; this procedure is stepped over the entire spectrum, producing the three-dimensional plots shown in Fig. 3. The ordinate, in scaled units, corresponds to the central energy of each rectangular filter. Our procedure is similar to that used in Ref. [19] for analyzing the time-frequency behavior of neon exposed to a fewcycle, $0.8 \mu \mathrm{m}$ sech pulse. Although the TDSE gives the photon energy directly for each harmonic order, for Fig. 3 the value of $I_{p}$ is subtracted from the photon energy to facilitate the comparison with the semiclassical electron return energy. Note that the time axis, in optical periods, begins $1 / 2$ cycle into the flat part of the pulse, when harmonic emission first becomes possible. Both the width and shape of the spectral filter were varied to insure consistent results; for Fig. 3 the rectangular filter width is $12.4 \mathrm{eV}$.

As can be seen in Fig. 3(a) the TDSE results for $2 \mu \mathrm{m}$ closely match the semiclassical prediction for the 1 st, $2 \mathrm{nd}$, 3 rd, and 4th electron returns. As the emission evolves from one $1 / 2$ cycle to the next a complicated structure develops due to the interference of the many contributing trajectories, but the role of the higher-order returns remains clear. The $0.8 \mu \mathrm{m}$ result also shows agreement with the classical first return, but the subsequent electron dynamics are more diffuse than in the long wavelength case and it is difficult to identify the multiple returns. It is not surprising that the time-dependent harmonic emission at $0.8 \mu \mathrm{m}$ deviates from the semiclassical expectation since the electron distribution shown in Fig. 1(a) is also inconsistent with this picture. However, the calculations are seen to coalesce around the classical result as the wavelength is increased, achieving the stunning agreement shown in Fig. 3(a). Surprisingly the harmonic emission localized on the higher-order returns has an unexpected large amplitude. Clearly the wave packet spread has been mitigated, possibly by Coulomb focusing [20] or trapping of electron population in excited states [21]. Although the higherorder returns become surprisingly more important at longer wavelengths (possibly affecting the wavelength scaling discussed above), phase matching may alter these conclusions.

Further analysis of Fig. 3 provides quantitative evidence for the scaling of the attosecond chirp with longer wavelength excitation. As described above, the absolute value of 

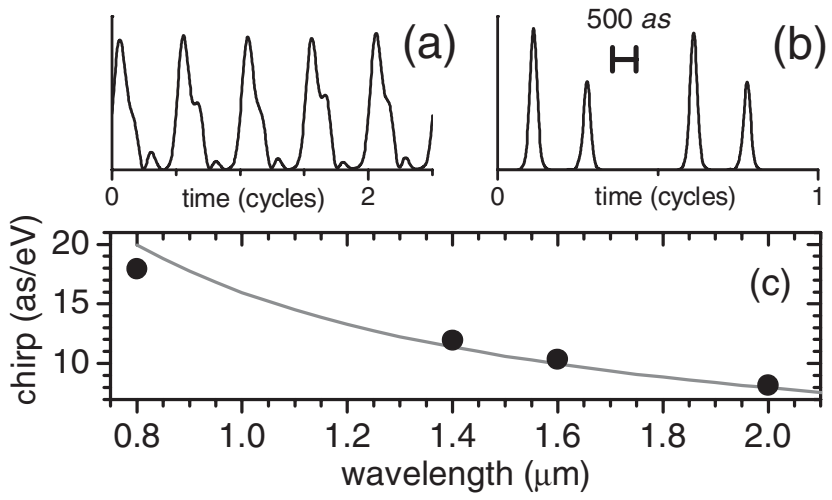

FIG. 4. Representative pulse trains for driving wavelengths of (a) 0.8 and (b) $2 \mu \mathrm{m}$ using the optimum bandwidths of 24.8 and $37.2 \mathrm{eV}$, respectively. (c) TDSE chirp (O) of the $\tau_{1}$ trajectory in argon as a function of wavelength for an intensity of $0.16 \mathrm{PW} / \mathrm{cm}^{2}$. Solid line is the classical $\lambda^{-1}$ prediction.

the chirp across successive harmonic orders (not within a single order) is expected to scale as $T / U_{p}$, which decreases as $\lambda^{-1}$. The slopes of the lines plotted in Fig. 3 are related to this wavelength-dependent chirp, which is shown for the argon "short" $\tau_{1}$ trajectory (positive chirp) in Fig. 4(c) for an intensity of $0.16 \mathrm{PW} / \mathrm{cm}^{2}$. The chirp derived from TDSE results closely matches the semiclassical scaling for all wavelengths and intensities investigated, but improved agreement is always achieved for longer wavelengths. The enhanced validity of classical dynamics for longer wavelength excitation along with the reduced attochirp defines a path towards "cleaner" and shorter attosecond bursts, as illustrated by the representative pulse trains shown for 0.8 and $2 \mu \mathrm{m}$ in Fig. 4 . These pulse trains, centered at energy $2.24 U_{p}(37.2 \mathrm{eV})$ for $0.8 \mu \mathrm{m}$ and $2.71 U_{p}(177 \mathrm{eV})$ for $2 \mu \mathrm{m}$, show more than one asec pulse per half cycle due to the contributions of multiple electron trajectories; phase matching should allow for the selection of a single asec burst per half cycle of the driving laser field. The attochirp is also reduced with increasing intensity, such that argon excited by a $2 \mu \mathrm{m}, 0.2 \mathrm{PW} / \mathrm{cm}^{2}$ pulse yields a minimum $\tau_{1}$ chirp of $6.59 \mathrm{as} / \mathrm{eV}$, corresponding to an optimum bandwidth of $42 \mathrm{eV}$ or 34 harmonics. For this bandwidth the pulse width extracted from the TDSE calculation in the time domain is 110 as, only slightly above the Fourier limit of 98 asec. If appropriate means can be found to compensate for the chirp, pulses well below 100 asec can be formed.

In conclusion, we have studied the wavelength scaling of the single atom strong-field response using both TDSE and SFA methods. The quantum simulations confirm the $\lambda^{2}$-scaling of the cutoff energy and the potential for producing $\mathrm{keV}$ electrons and photons from longer wavelength lasers. In addition, there are some surprising results: first, the scaling of the HHG yield $\left(\lambda^{-6}\right)$ at constant intensity has been shown to be different from the generally accepted $\lambda^{-3}$. Second, the TDSE results show an unexpectedly strong contribution to HHG from higher-order returns of the electron wave packet. Finally, the attosecond chirp across successive harmonics decreases as $\lambda^{-1}$, leading to the possibility of generating extremely short attosecond $\mathrm{x}$-ray pulses with midinfrared wavelengths.

We thank P. Johnsson, J. Mauritsson, and W. Becker for valuable discussions. This work was performed with support from DOE/BES under Contract No. DE-FG0204ER15614 and computational resources from the Ohio Supercomputer Center. T. A. and P.S. acknowledge EU support under Contract No. MCRTN-CT-2003-505138.

[1] L. V. Keldysh, Sov. Phys. JETP 20, 1307 (1965).

[2] K. J. Schafer, Baorui Yang, L.F. DiMauro, and K.C. Kulander, Phys. Rev. Lett. 70, 1599 (1993).

[3] P. B. Corkum, Phys. Rev. Lett. 71, 1994 (1993).

[4] M. Hentschel et al., Nature (London) 414, 509 (2001).

[5] I. J. Sola et al., Nature Phys. 2, 319 (2006).

[6] F. Lindner et al., Phys. Rev. Lett. 95, 040401 (2005).

[7] T. Fuji et al., Opt. Lett. 31, 1103 (2006).

[8] K. C. Kulander, Phys. Rev. A 36, 2726 (1987).

[9] M. Lewenstein et al., Phys. Rev. A 49, 2117 (1994).

[10] W. Becker, S. Long, and J. K. McIver, Phys. Rev. A 50, 1540 (1994).

[11] H. G. Muller, Phys. Rev. A 60, 1341 (1999).

[12] Y. Mairesse et al., Science 302, 1540 (2003).

[13] K. Burnett, V. C. Reed, J. Cooper, and P. L. Knight, Phys. Rev. A 45, 3347 (1992).

[14] B. Walker, B. Sheehy, K. C. Kulander, and L. F. DiMauro, Phys. Rev. Lett. 77, 5031 (1996).

[15] B. Shan and Z. Chang, Phys. Rev. A 65, 011804(R) (2001).

[16] G. L. Yudin and M. Y. Ivanov, Phys. Rev. A 64, 013409 (2001).

[17] A. Gordon and F.X. Kartner, Opt. Express 13, 2941 (2005).

[18] M.B. Gaarde and K. J. Schafer, Phys. Rev. A 65, 031406(R) (2002).

[19] V.S. Yakovlev and A. Scrinzi, Phys. Rev. Lett. 91, 153901 (2003).

[20] G. L. Yudin and M. Y. Ivanov, Phys. Rev. A 63, 033404 (2001).

[21] A. Pukhov, S. Gordienko, and T. Baeva, Phys. Rev. Lett. 91, 173002 (2003). 